\title{
Numerical Modelling of a Graphene - Resonant Tunneling Diode as a High Frequency Oscillator
}

\author{
Shakirudeen Lasisi* , Prof Trevor Benson, Dr Gabriele Gradoni, Dr Kristof Cools, Dr Mark Greenaway
}

\section{Background}

New solid-state sources of $\mathrm{GHz}-\mathrm{THz}$ electromagnetic (EM) radiation continue to have many applications in high-speed electronics, communications, security, and medicine. Using Graphene-based materials is particularly promising due to its high electron mobility and configurability of the device structures. However, modelling its electromagnetic behaviour computationally along with the inherent complexities of the device (e.g. non-linearities, and quantum effects) can be quite challenging using current tools.

\section{Our Approach}

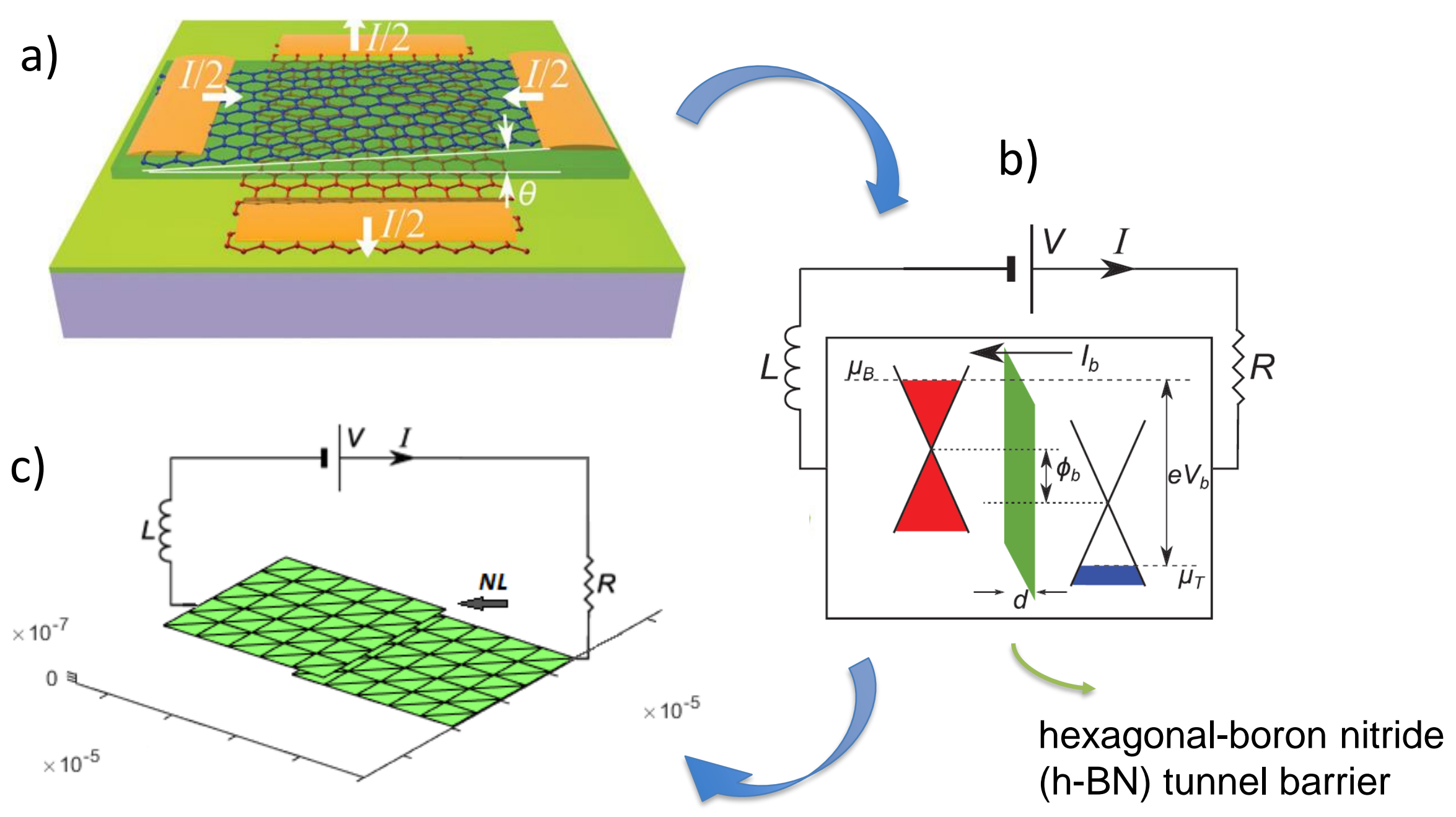

Figure1: showing (a) Experimental model of the GRTD device [1] (b) Schematic diagram of the tank circuit and band diagram of the tunnel region [1] (c) Approximate model used in the EM simulation (Ground plane not shown in figure).

We use a simplified approximate model (fig 1 ) to reduce the complexity of the oscillator's structure and derive formulations that describe its electromagnetic and intrinsic behaviours. In this poster, we report new formulations, finite element spaces and general progress in modelling a graphene resonant tunnelling diode (GRTD) using the Time-domain boundary element method (TD-BEM). We also explore the possibility of mutual coupling and synchronization between two GRTD devices.

\section{Formulation}

Consider the configuration illustrated in fig 1c above. In order to generate self-sustained oscillations, the device which exhibits nondifferential conductance is placed in a resonant circuit.

$$
\begin{gathered}
Z^{0} I^{k}+V N^{k}+V R^{k}+V L^{k}=-V 0^{k}-\sum_{l=1}^{k-1} Z^{(k-l)} I^{l}(1) \\
Z_{n m}^{(k-l)}=-c \eta \int_{\Gamma} \nabla \cdot f_{n} \int_{\Gamma} \frac{\nabla^{\prime} \cdot f_{m} T^{(k-l)}}{4 \pi R} d S^{\prime} d S-\eta \int_{\Gamma} f_{n} \int_{\Gamma} \frac{f_{m} \partial_{t}^{2} T^{(k-l)} d t}{4 \pi c R} d S^{\prime} d S
\end{gathered}
$$

Using a time-domain BEM formulation we can compute the current density induced on the plates of the device by coupling the marchingon-in-time (MOT) equation (eqn 1) with relevant circuit equations. Note: The non-local dynamical conductivity and quantum capacitance (among others) found in graphene are not yet included in the model.

\section{Basis Function}

a)

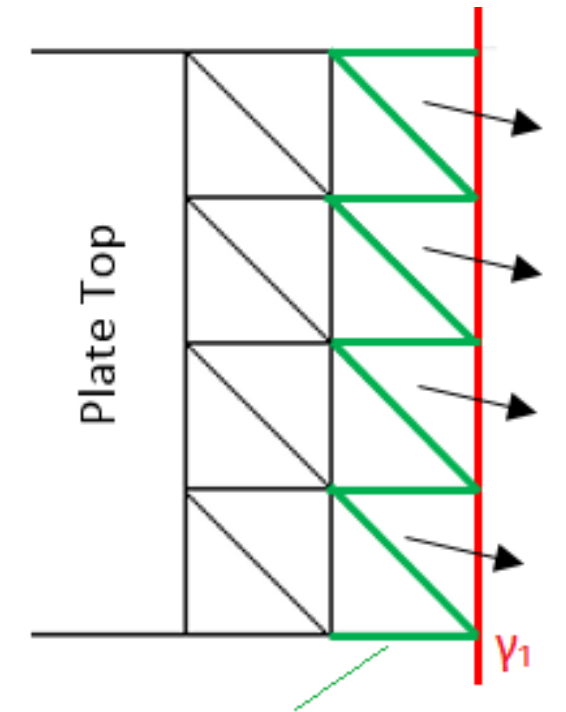

half RWG

Figure 2: Illustrating (a) Current conservation between ports on both plates [2] (b) Alternating current to account for variations along port [2]

To define the tunnel current between the plates, an enriched RaoWilton-Glisson (RWG) basis [2] is used to discretise the current as in eqn (2). Fig 2 illustrates the finite element space defined at the edges of the plates.

$$
\boldsymbol{j}=\sum a^{i}(t) \boldsymbol{f}^{i}+\sum a^{v}(t) \boldsymbol{f}^{v}+\sum a^{c}(t) \boldsymbol{f}^{c}
$$

\section{High Frequency Generation}

a)

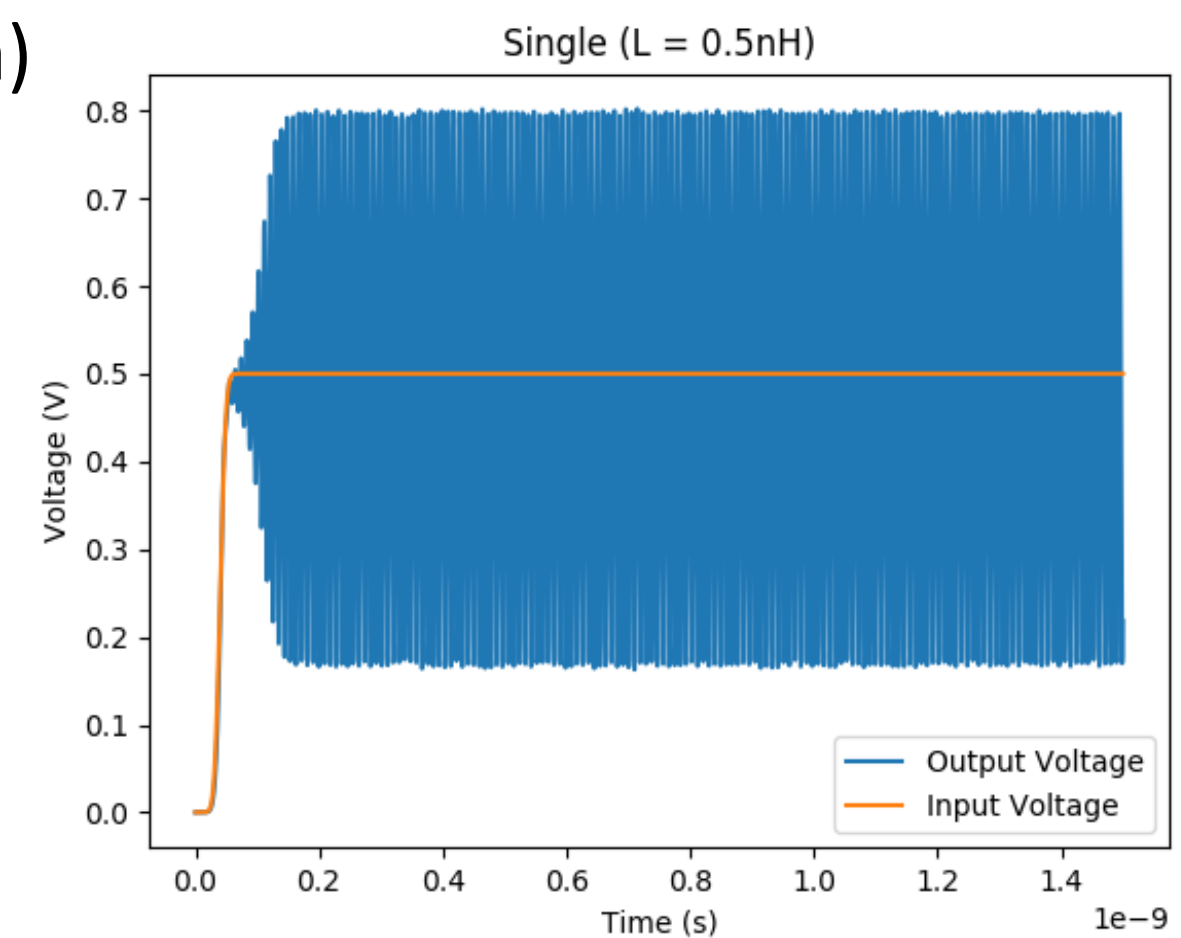

Figure 3: shows (a) Output voltage oscillations (b) Spectrum of Output signal (c) Non-linear I-V characteristic between plates.

Given a lumped inductance of $L=0.5 \mathrm{nH}$, negative resistance of $R_{n}=1.19 \mathrm{k} \Omega$ and series resistance of $R=59.5 \Omega$, self-sustained oscillations of fundamental frequency $f=108 \mathrm{GHz}$ can be observed.

In other configurations, oscillations on the higher end of the $\mathrm{GHz}$ range have been recorded.

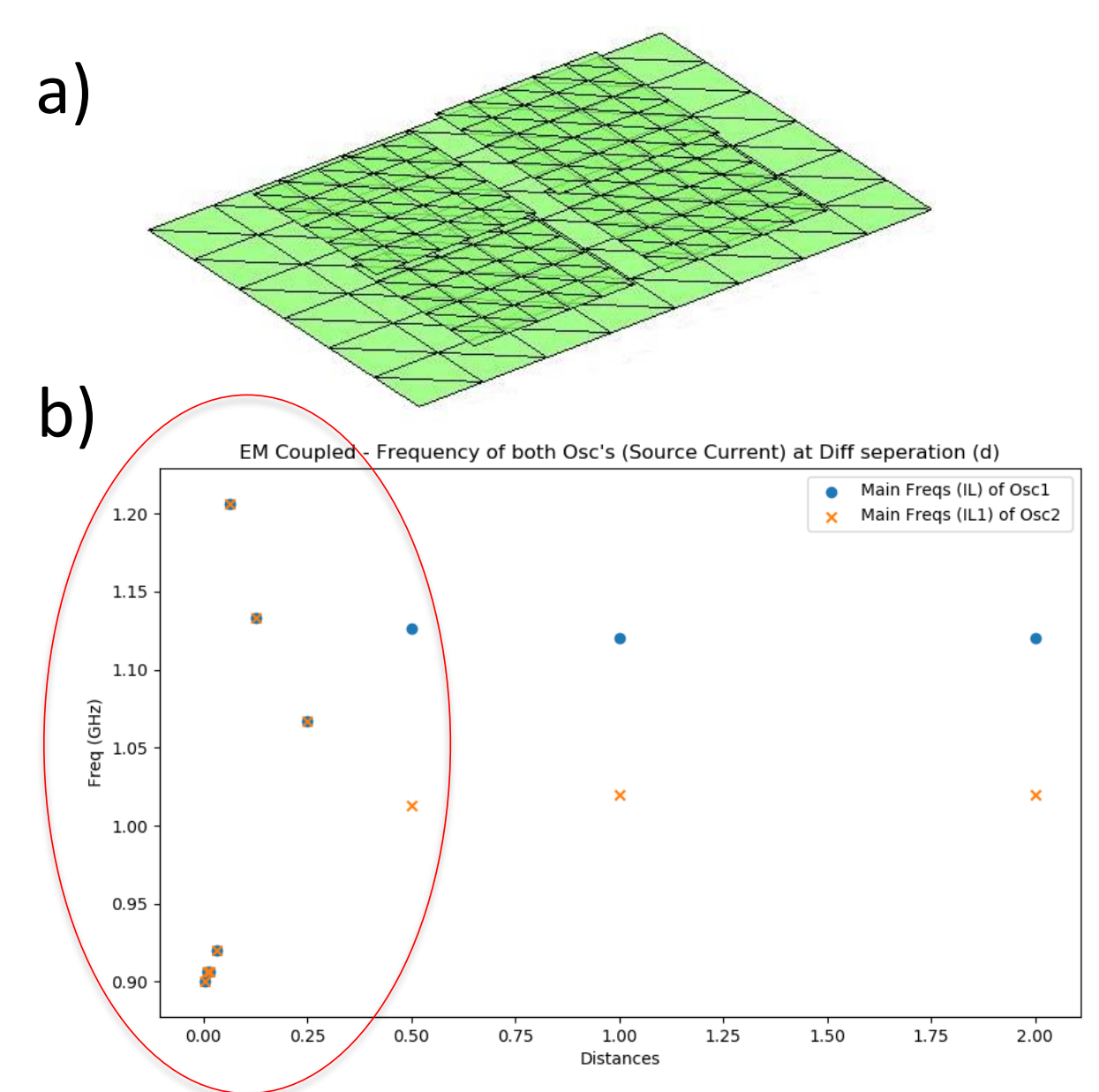

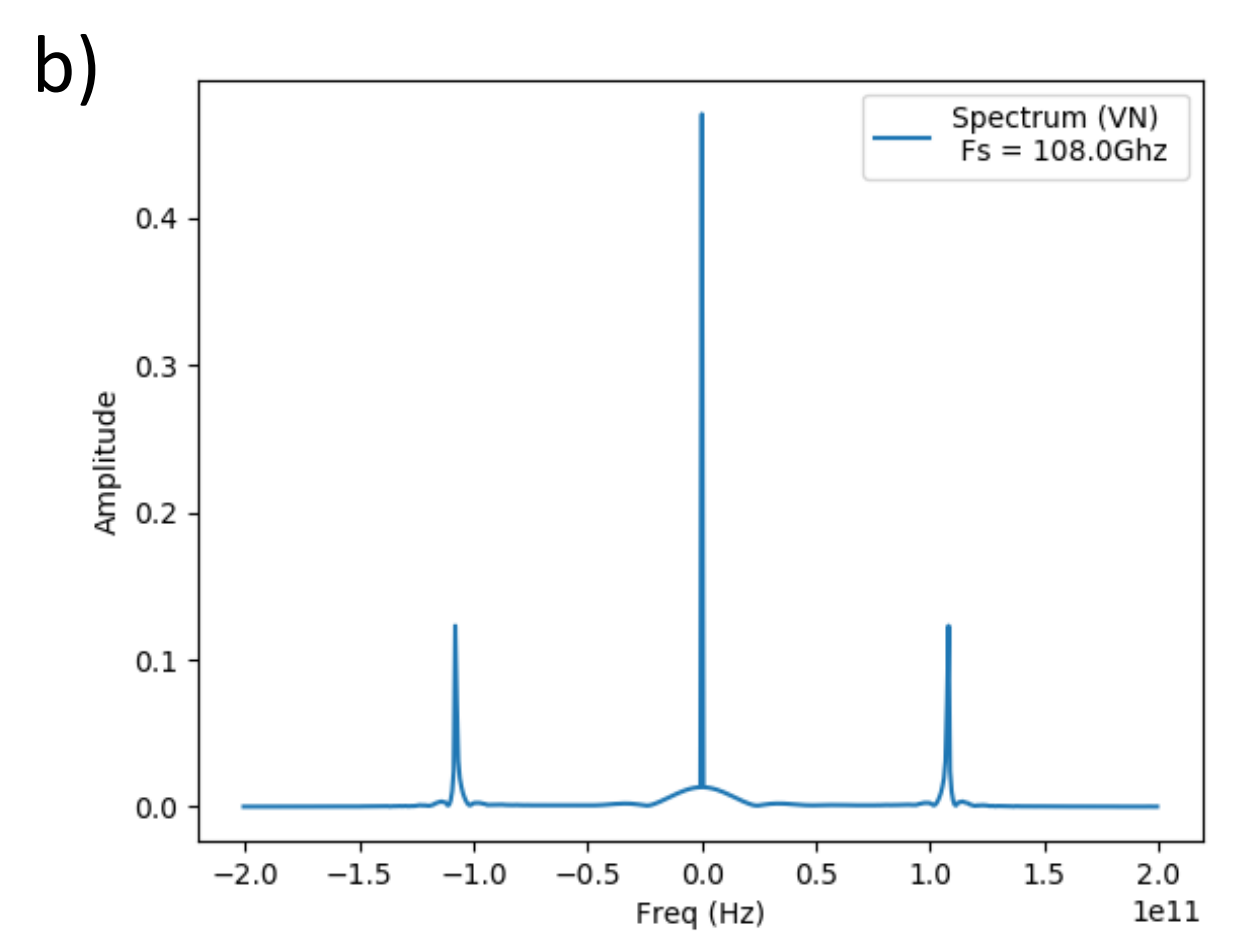

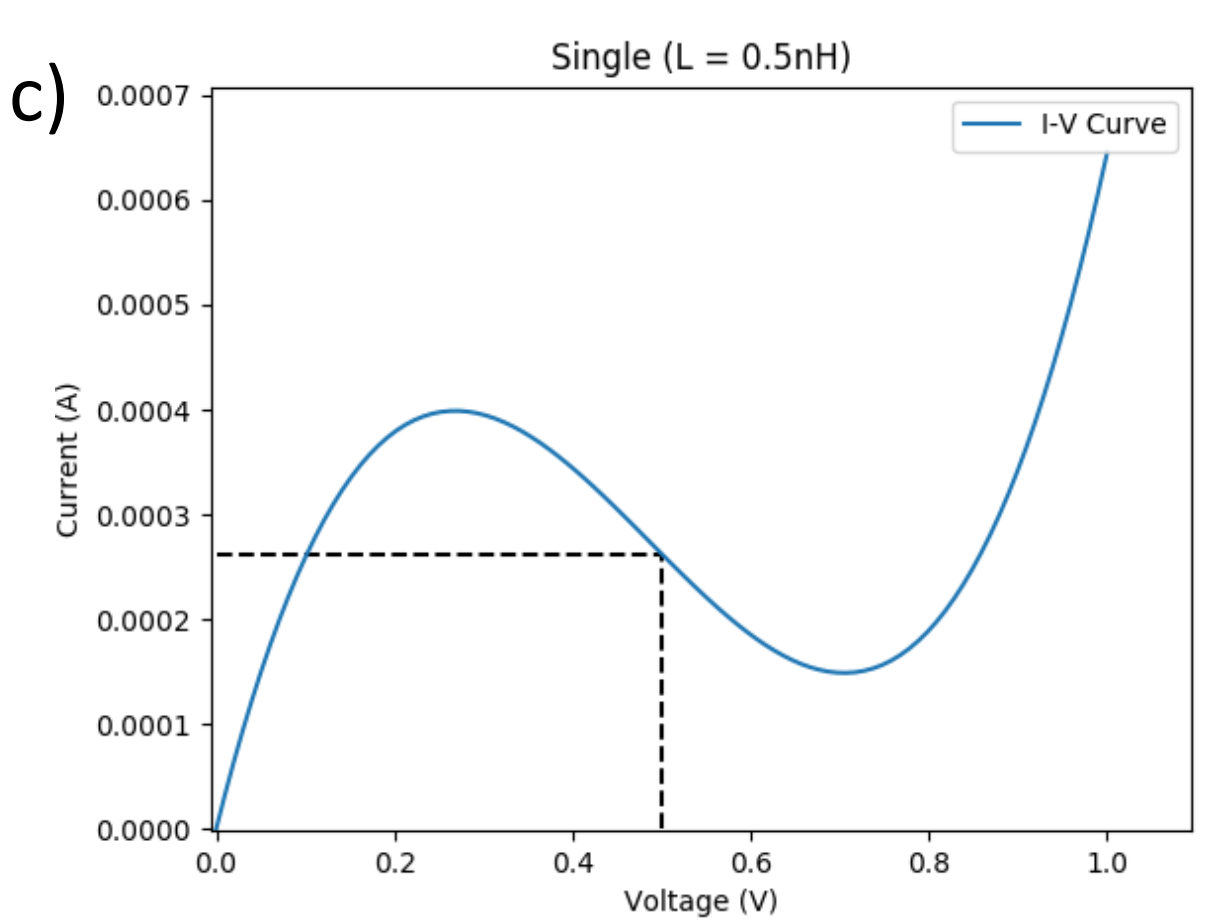

Synchronisation

In a different simulation, depending on the frequency of the individual oscillators, the bias voltage, and separation distance of both devices, we find that the frequencies of both oscillators lock at a single frequency even when the individual frequencies differ by a significant margin. See fig $4 a$ and $4 b$ to the left.

Figure 4 (left): shows synchronisation when two oscillators are separated at $d \leq 0.25 \lambda$

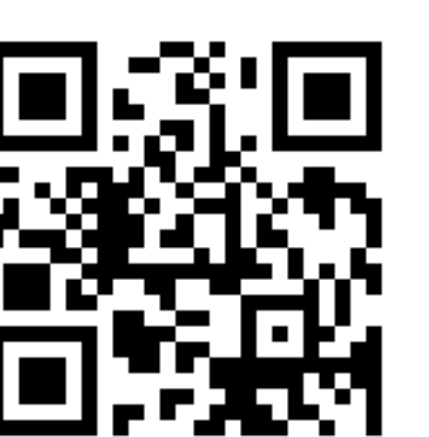
[1] 